\title{
The incidence of type 1 diabetes is increasing in both children and young adults in Northern Italy: 1984-2004 temporal trends
}

\author{
G. Bruno • G. Novelli • F. Panero • M. Perotto • \\ F. Monasterolo • G. Bona • A. Perino • I. Rabbone • \\ P. Cavallo-Perin • F. Cerutti • \\ Piedmont Study Group for Diabetes Epidemiology
}

Received: 8 May 2009 /Accepted: 3 August 2009 /Published online: 11 October 2009

(C) Springer-Verlag 2009

\begin{abstract}
Aims/hypothesis A shift towards younger age at onset of diabetes in susceptible people has been suggested as a possible explanation for the increasing temporal trend in incidence of type 1 diabetes. We aimed to test this hypothesis by assessing trends in incidence rates in the period 1984-2004 in children and young adults in Northern Italy.

Methods The study bases were: (1) children resident in the Province of Turin in the period 1984-2004 and in the remaining areas of the Piedmont Region in the period 1990-2004; and (2) young adults (15-29 years) resident in the Province of Turin in the period 1984-2003. Temporal trends in rates were analysed using Poisson regression models.
\end{abstract}

Electronic supplementary material The online version of this article (doi:10.1007/s00125-009-1538-x) contains supplementary material, which is available to authorised users. This includes a list of the participants of the Piedmont Study Group for Diabetes Epidemiology.

G. Bruno $(\bowtie) \cdot G$. Novelli $\cdot$ F. Panero $\cdot$ M. Perotto $•$

F. Monasterolo $\cdot$ P. Cavallo-Perin

Department of Internal Medicine, University of Turin,

corso Dogliotti 14,

10126 Turin, Italy

e-mail: graziella.bruno@unito.it

G. Bona $\cdot$ A. Perino

Department of Pediatrics, University of Novara,

Novara, Italy

I. Rabbone $\cdot$ F. Cerutti

Department of Pediatrics, University of Turin,

Turin, Italy
Results A total of 1,773 incident cases were identified. Overall incidence rates/100,000 person-years in the age groups $0-14$ and $15-29$ years were 11.3 (95\% CI $10.7-$ $12.0)$ and 7.1 (95\% CI 6.6-7.7), respectively, with sex differences among young adults only (incidence rate ratio [IRR] in males vs females 1.41 [95\% CI 1.20-1.64]). Average annual increases in incidence rates were similar in children and young adults at 3.3\% (95\% CI 2.5-4.1). Compared with the period 1984-89, in 2000-2004 a 60\% higher risk was found in both age $0-14$ years (IRR 1.60, 95\% CI 1.31-1.95) and 15-29 years (IRR 1.57, 95\% CI 1.26-1.96) groups. The Poisson modelling showed no interaction between calendar period and age at onset.

Conclusions/interpretation Incidence of type 1 diabetes in Northern Italy is increasing over time in both children and young adults, not supporting the hypothesis of a shift towards younger age as the main explanation for the increasing temporal trend in children.

Keywords Adult-onset diabetes · Incidence - Italy · Registry Survey $\cdot$ Temporal trend

\section{Abbreviations \\ IRR Incidence rate ratio \\ LR Likelihood ratio}

\section{Introduction}

Geographical and temporal variations in the incidence of type 1 diabetes have been pointed out worldwide, with recent data from Northern European registries showing that risk is increasing even faster than before, particularly in Finland [1-3]. A shift towards younger age at onset in 
susceptible individuals rather than a true lifetime increasing risk of the disease has been suggested by the few studies that extended the recruitment of incident cases up to adults $[4,5]$.

The first Italian population-based registry of type 1 diabetes was set up in 1984 in Turin, Northern Italy, to monitor the incidence rate of the disease up to age 29 years [6]. In this report, we aimed to extend the study base of our registry over both space and time, to examine incidence trends of the disease in both children ( $0-14$ years) and young adults (15-29 years) in the period 1984-2004.

\section{Methods}

The study bases of this report were: (1) children 0-14 years of age resident in the Province of Turin (number of inhabitants 2,165,619 at the 2001 census) in the period 1984-2004 and in the remaining areas of the Piedmont Region (number of inhabitants 2,049,058) in the period 1990-2004; and (2) young adults (15-29 years) resident in the Province of Turin in the period 1984-2003. Incident cases of type 1 diabetes arising in the study period were identified through the following sources of ascertainment: (1) diabetes clinics from public and private hospitals, to which diabetic patients are referred after diagnosis, (primary source); and (2) files of all patients who had obtained exemption from payment for drugs, syringes and glucosemonitoring strips because of a diagnosis of diabetes mellitus (secondary source). The estimated completeness of ascertainment was assessed by using the two-sample capture-recapture method. A diagnosis of type 1 diabetes was based on permanent insulin treatment within 6 months of diagnosis, fasting C-peptide levels $\leq 0.20 \mathrm{nmol} / 1$ or positivity for antibodies to islet cells (ICA) or GADA. Denominators of incidence rates were intercensual estimates of residents in the Piedmont Region and its eight provinces (Turin, Alessandria, Asti, Biella, Cuneo, Novara, Verbania and Vercelli). Incidence rates for the periods 1984-1989, 1990-1994, 1995-1999 and 2000-2004 were compared by computing incidence rate ratios (IRRs) and 95\% CIs.

Time trends in incidence rates were modelled by Poisson regression modelling. Sex, age (six 5 year age groups), province $(n=8)$ and calendar period (1984-1989, 19901994, 1995-1999 and 2000-2004) were independent variables. Models were compared by the likelihood ratio (LR) test. We also estimated time trends after stratification by age group to assess if they differed significantly. Ageperiod cohort analysis was also performed, updating previous data from the Province of Turin, as previously described [6]. All statistical analyses were performed using STATA, release 10.0 (Stata, College Station, TX, USA).

\section{Results}

In the period 1984-2004, 1,773 incident cases of type 1 diabetes aged 0-29 years were identified in the study base, giving an overall incident rate/100,000 person-years of 9.3 (95\% CI 8.9-9.7), with an high estimated completeness of ascertainment over calendar periods 1984-1989, 1990-1994, 1995-1999 and 2000-2004, in the age group $0-14$ years $(99 \%, 97 \%, 99 \%$ and $99 \%)$ and in the age group $15-29$ years $(95 \%, 99 \%, 98 \%$ and $97 \%)$. Rates are based on 1,123 children identified in the period 1984-2004 and on 650 young adults identified in the period 1984-2003.

Overall incidence rates/100,000 person-years were 11.3 (95\% CI 10.7-12.0) in the age group $0-14$ years and 7.1 (95\% CI 6.6-7.7) in the age group 15-29 years. Rates/ 100,000 person-years in the age groups $0-4,5-9$ and $10-$ 14 years were 8.3 (95\% CI 7.3-9.4), 11.7 (95\% CI 10-613.0) and 13.6 (95\% CI 12.4-14.8), respectively; indeed, the majority of incident cases were identified in the pubertal age (IRR 1.64, 95\% CI 1.41-1.91, with respect to the age group $0-4$ years, $p<0.0001$ ). Incidence rates by age at onset and cumulative incidence by calendar period are shown in the Electronic supplementary material (ESM) Figs 1 and 2.

The risk of childhood diabetes was similar in males and females: $11.8 / 100,000$ (95\% CI 10.9-12.8) vs $10.8 / 100,000$ (95\% CI 9.9-11.8), respectively. In contrast, a 40\% higher risk in males than in females was evident among young adults: 8.2/100,000 (95\% CI 7.1-9.1) vs 5.9/100,000 (95\% CI 5.2-6.6), giving an IRR of 1.41 (95\% CI 1.20-1.65).

The incidence rate increased over the period 1984-2004, with an average annual increase of 3.3\% (95\% CI 2.5-4.1), slightly higher in age $0-14$ years $(4.3 \%, 95 \%$ CI $2.0-6.6)$ than in age $15-29$ years $(2.8 \%, 95 \%$ CI $1.0-4.6)$. Indeed, the largest annual increase was seen in the $0-4$ year age group (4.3\%, 95\% CI 2.0-6.6); corresponding values in the age groups $5-9$ and $10-14$ years were $2.8 \%$ (95\% CI 1.0 4.6 ) and $2.7 \%$ (95\% CI 1.2-4.3\%). However, differences in annual increases among age groups did not reach statistical significance.

Incidence rates $/ 100,000$ person-years separately for age group, calendar period and area are shown in Table 1. In the last time period, a $60 \%$ increased risk with respect to the period 1984-1989 was found in both age 0-14 years (IRR $1.60,95 \%$ CI 1.31-1.95) and 15-29 years (IRR 1.57, 95\% CI 1.26-1.96). Point estimate of risks were twofold higher in the latter time period in the age group 0-4 years (IRR $1.92,95 \%$ CI $1.21-3.05$ ) and in the age group 5-9 years (IRR 1.90, 95\% CI 1.35-2.68), but differences were not statistically significant. Examining incidence rates in children resident in the whole Piedmont Region in the period 1990-2004, the amount of the increase in the period 2000-2004 was 33\% with respect to 1990-1994. 


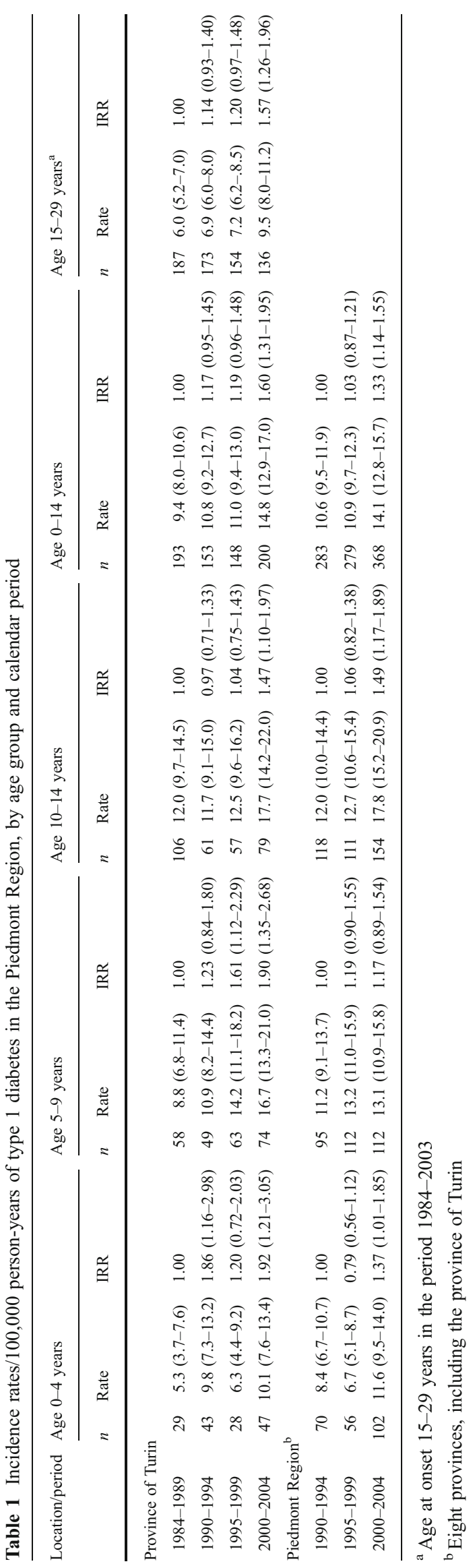


Poisson regression analysis (Table 2) showed a linearly increasing trend over time, independently of age, sex and geographical area (model 5). The Poisson modelling showed no evidence of interaction between calendar period and either sex (model 6) or age (model 7), whereas interaction between age and sex was significant (model 8; $p=0.01$ ); in this final model, the linearly increasing trend was $3.0 \%$ per year $(95 \%$ CI $2.1-3.8)$.

Age-period cohort analysis (ESM Tables 1 and 2) showed a linearly increasing trend (drift), not interpretable on a statistical basis as related to calendar period or cohort effects, which corresponds to an IRR of 1.17 (1.13-1.21; $p<0.0001$ ) for each age span of 5 years.

\section{Discussion}

Our study shows that the incidence rate of type 1 diabetes in Northern Italy has markedly increased over the monitored time period of 20 years. Compared with the period 1984-1989, the risk had increased by $60 \%$ in the period $2000-2004$. The amount of the annual increase (3.3\%) is similar to that found in other European areas. The new finding of our analyses is that the increasing temporal trend is similarly evident in children and young adults. Therefore, our data are not consistent with the hypothesis of a shift towards a younger age at onset in susceptible people as a possible explanation for the temporal increase of childhood diabetes, as suggested by some $[4,5]$ but not all registries [7, 8]. Our data expand current knowledge on this issue to the Mediterranean population, suggesting that ubiquitous environmental deter- minants affecting both children and young adults born in the last three decades are involved in this phenomenon.

The identification of widespread determinants of unknown origin is a difficult task for epidemiological research. Although the increasing temporal trend seems to be even faster in more recent years in Germany, Austria and Finland, no spike in incidence has been identified in these countries [1-3]. As recently pointed out, viral infections could be involved in the pathogenesis of diabetes by inhibiting rather than inducing a diabetogenic response [9]. Studies have proposed that the decreasing early life exposure to infectious diseases, which typically occurred over the past three decades in developed countries, could be involved in the increasing temporal trend of immunemediated disorders, multiple sclerosis, type 1 diabetes and inflammatory bowel diseases [10]. All of these diseases, by contrast, are uncommon in developing countries. Further epidemiological and immunological studies, however, are needed to test the so-called 'hygiene hypothesis'.

Consistently with previous age-period cohort analysis of the registry of the Province of Turin in the period 1984-1996 [6], in this report we showed a linearly increasing trend, which could be attributed to either a period or a cohort effect. Our data are based on the extension of our registry to residents in the whole Piedmont Region, thus allowing a description of risk of type 1 diabetes on a population of almost 5 million inhabitants over a 20 year time period. The long time span examined in this report, the almost complete case ascertainment over all the study period and the recruitment of incident cases arising both in childhood and in adulthood with accurate methodology are strengths of the present report.

Table 2 Result of Poisson regression model fitting for type 1 diabetes in the Piedmont Region, 1984-2004

\begin{tabular}{|c|c|c|c|c|c|}
\hline Model/variables & Deviance & $d f$ & LR $\chi^{2}$ for the last term & $d f$ & $p$ value \\
\hline 1 Age & 974.73 & 870 & 143.5 & 5 & $<0.0001$ \\
\hline 2 Age + sex & 960.20 & 869 & 14.1 & 1 & 0.0002 \\
\hline 3 Age + sex + year & 913.72 & 868 & 46.9 & 1 & $<0.0001$ \\
\hline 4 Age + sex + period & 911.68 & 866 & $48.95^{\mathrm{a}}$ & $3^{\mathrm{a}}$ & $<0.0001$ \\
\hline 5 Age + sex + year + province & 901.50 & 861 & $12.23^{\mathrm{b}}$ & $7^{\mathrm{b}}$ & 0.09 \\
\hline 6 Age + sex + year + province + age $_{2} \times$ period & 897.22 & 856 & 4.27 & 5 & 0.52 \\
\hline 7 Age + sex + year + province + sex $\times$ period & 895.06 & 856 & $6.44^{\mathrm{c}}$ & $5^{\mathrm{c}}$ & 0.27 \\
\hline 8 Age + sex + year + province + age $_{2} \times$ sex & 895.02 & 860 & $6.48^{\mathrm{c}}$ & $1^{\mathrm{c}}$ & 0.01 \\
\hline
\end{tabular}

Age is entered in models as six age groups $(0-4,5-9,10-14,15-19,20-24,25-29$ years)

$\mathrm{Age}_{2}$ is entered in models as age $0-14$ and 15-29 years

Year is entered in models as a linear term of year at onset

Period is entered in models as four calendar periods of onset (1984-1989, 1990-1994, 1995-1999, 2000-2004)

Province is entered in models as eight provinces (Turin, Alessandria, Asti, Biella, Cuneo, Novara, Verbania, Vercelli)

${ }^{\text {a }}$ With respect to model 2

${ }^{\mathrm{b}}$ With respect to model 3

${ }^{\mathrm{c}}$ With respect to model 5 
Monitoring incidence of type 1 diabetes through the registries is both a time- and resource-consuming activity. However, population-based data are still needed, both to suggest hypotheses on the determinants of the disease and to provide data on its global burden for healthcare planners. Since a doubling in the number of incident cases can be expected every three decades, both quantity and quality of care provided to young people with diabetes should be markedly improved over the coming years.

In conclusion, incidence of type 1 diabetes in Northern Italy is increasing over time in both children and young adults. Our findings do not support the hypothesis of a shift towards younger age at onset as the main explanation for the increasing temporal trend of the disease in children.

Acknowledgements This study was supported by grants from the Piedmont Region (Ricerca Sanitaria Finalizzata 2007 and 2008) and from the University of Turin.

Duality of interest The authors declare that there is no duality of interest associated with this manuscript.

\section{References}

1. Ehehalt S, Blumenstock G, Willasch AM et al (2008) Continuous rise in incidence of childhood Type 1 diabetes in Germany. Diabet Med 25:755-757
2. Schober E, Rami B, Waldhoer T, Austrian Diabetes Incidence Study Group (2008) Steep increase of incidence of childhood diabetes since 1999 in Austria. Time trend analysis 1979-2005. A nationwide study. Eur J Pediatr 167:293-297

3. Harjutsalo V, Sjöberg L, Tuomilehto J (2008) Time trends in the incidence of type 1 diabetes in Finnish children: a cohort study. Lancet 371:1777-1782

4. Pundziute-Lyckå A, Dahlquist G, Nyström L et al (2002) The incidence of Type 1 diabetes has not increased but shifted to a younger age at diagnosis in the 0-34 years group in Sweden 1983 to 1992. Diabetologia 45:783-791

5. Weets I, de Leeuw IH, Du Caju MVL et al (2002) The Belgian Diabetes Register: the incidence of type 1 diabetes in the age group 0-39 years has not increased in Antwerp (Belgium) between 1989 and 2000. Evidence for earlier disease manifestation. Diabetes Care 25:840-846

6. Bruno G, Merletti F, Biggeri A et al (2001) Increasing trend of type 1 diabetes in children and young adults. Analysis of age, period and birth cohort effect during 1984-96. Diabetologia 44:22-25

7. Lammi N, Blomstedt PA, Moltchanova E, Eriksson JG, Tuomilehto J, Karvonen M (2008) Marked temporal increase in the incidence of type 1 and type 2 diabetes among young adults in Finland. Diabetologia 51:897-899

8. Pundziute-Lyckå A, Urbonaite B, Ostrauskas R, Zalinkevicius R, Dahlquist GG (2003) Incidence of type 1 diabetes in Lithuanians aged $0-39$ years varies by the urban-rural setting, and the time change differs for men and women during 1991-2000. Diabetes Care 26:671-676

9. Von Herrath M (2009) Can we learn from viruses how to prevent type 1 diabetes? The role of viral infections in the pathogenesis of type 1 diabetes and the development of novel combination therapies. Diabetes 58:2-11

10. Cooke A (2009) Review series on helminths, immune modulation and the hygiene hypothesis: how might infection modulate the onset of type 1 diabetes? Immunology 126:12-17 